\title{
Of Brains and Computers
}

\author{
Jan M. Rabaey \\ Professor in the Graduate School, UC Berkeley \\ CTO STCO Division, IMEC, Belgium
}

\begin{abstract}
:
The human brain - which we consider to be the prototypal biological computer - in its current incarnation is the result of more than a billion years of evolution. Its main functions have always been to regulate the internal milieu and to help the organism/being to survive and reproduce. With growing complexity, the brain has adapted a number of design principles that serve to maximize its efficiency in performing a broad range of tasks. The physical computer, on the other hand, had only 200 years or so to evolve, and its perceived function was considerably different and far more constrained that is to solve a set of mathematical functions. This however is rapidly changing. One may argue that the functions of brains and computers are converging. If so, the question arises if the underlying design principles will converge or cross-breed as well, or will the different underlying mechanisms (physics versus biology) lead to radically different solutions.
\end{abstract}

\section{CCS Concepts/ACM Classifiers}

- Hardware $\rightarrow$ Emerging technologies

\section{Author Keywords}

Brain, computation, design convergence, neural design

\section{Bio:}

Jan is a Professor in the Graduate School in the EECS Department at the University of California at Berkeley, where he held the Donald O. Pederson Distinguished Professorship for over 30 years before retiring. Before joining the faculty at
UC Berkeley, he was a research manager at IMEC from 1985 until 1987. He is a founding director of the Berkeley Wireless Research Center (BWRC) and the Berkeley Ubiquitous SwarmLab, and has served as the Electrical Engineering Division Chair at Berkeley twice. In 2019, he also became the CTO of the SystemTechnology Co-Optimization (STCO) Division of IMEC, Belgium.

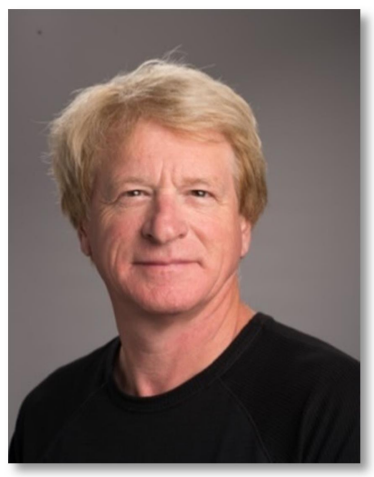

Prof. Rabaey has made high-impact contributions to a number of fields, including advanced wireless systems, low power integrated circuits, mobile devices, sensor networks, and ubiquitous computing. His current focus is the interaction between the cyber and the biological world (amongst many other things).

$\mathrm{He}$ is the recipient of major awards, amongst which the IEEE Mac Van Valkenburg Award, the European Design Automation Association (EDAA) Lifetime Achievement award, the Semiconductor Industry Association (SIA) University Researcher Award, and the SRC Aristotle Award. He is an IEEE Fellow, a member of the Royal Flemish Academy of Sciences and Arts of Belgium, and has received a number of honorary doctorates. He has been involved in a broad variety of start-up ventures.

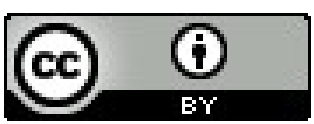

This work is licensed under a Creative Commons Attribution International 4.0 License.

ISPD '21, March 22-24, 2021, Virtual Event, USA.

(C) 2021 Copyright is held by the owner/author(s).

ACM ISBN 978-1-4503-8300-4/21/03. DOI: https://doi.org/10.1145/3439706.3446899 